Case Report

\title{
Low-grade fibromyxoid sarcoma: an unusual cardiac location
}

\author{
Amedeo Ferlosio, Elena Doldo, Patrizio Polisca, Augusto Orlandi* \\ Department of Biomedicine and Prevention, "Tor Vergata" University, Rome, Italy
}

\section{A R T I C L E I N F O}

\section{Article history:}

Received 19 September 2012

Received in revised form 2 November 2012

Accepted 27 November 2012

\section{Keywords:}

Cardiac sarcoma

Myxoid tumor

Low-grade fibromyxoid sarcoma

\begin{abstract}
A B S T R A C T
We report the unusual cardiac localization of a primary low-grade fibromyxoid sarcoma of the right ventricle in a 57-year-old woman. Histological examination revealed a prevalent myxoid appearance with whorling growth pattern of small or spindle cells with bland features alternating with rare more collagenous hypocellular areas with rare atypical cells. Genomic polymerase chain reaction of genomic DNA revealed the typical FUS/Creb3L2 fusion gene products typical of low-grade fibromyxoid sarcoma. The tumor was surgically removed and recurred after 7 years as high-grade pleomorphic sarcoma. The patient died 6 months after the clinical manifestation of recurrence. Low-grade fibromyxoid sarcoma of soft tissues is a rare, distinctive variant of fibrosarcoma-typically arising in deep soft tissue of lower extremities and trunk-that rarely metastasizes. Clinically, low-grade fibromyxoid sarcoma is characterized by a longer survival rate compared to other sarcomas, suggesting its consideration in the differential diagnosis of cardiac tumors with a myxoid appearance.
\end{abstract}

(c) 2013 Elsevier Inc. All rights reserved.

\section{Case description}

A 57-year-old woman was admitted for progressive signs of right cardiac failure. A two-dimensional echocardiogram revealed an endocavitary sessile cardiac mass of $5 \mathrm{~cm}$ in higher dimension attached to the right ventricular wall with a smooth surface, mimicking a cardiac myxoma. Total-body computer tomography exam did not reveal any other mass or metastasis, or any evident infiltrative pattern of the tumor. For the progressive symptoms of right cardiac failure, a surgical intervention was decided based on the clinical preoperative diagnosis. The tumor was removed through a median sternotomy. The surgical treatment required cardiopulmonary bypass and cardiac arrest by cardioplegic blood solution. Histological examination of the tumor (Fig. 1) revealed a prevalent myxoid appearance with whorling growth pattern of small or spindle cells with bland features alternating with collagenous and hypocellular ones and occasional cells with a lipoblast-like appearance. The vascularization consisted of arcades of small vessels and arterioles with perivascular fibrosis. Even in cellular areas, mitosis and atypia were extremely rare, although myocardial infiltration and large occasional hyperchromatic nuclei were observed. Tumor cells were immunopositive for vimentin and negative for cytokeratin, S-100,

Conflict of interest: The authors declare no conflict of interest. The study was conducted with A.O.'s personal research grant.

* Corresponding author. Institute of Anatomic Pathology, Department Biomedicine and Prevention, Tor Vergata University of Rome, Via Montpellier 1, 00133 Rome, Italy. Tel.: +39 6 20903960; fax: +39 620902209 .

E-mail address: orlandi@uniroma2.it (A. Orlandi). smooth muscle actin, desmin, and CD34. A diagnosis of low-grade fibromyxoid sarcoma (LGFMS) was made. The patient refused any other therapeutic regiment. After 7 years, the woman was readmitted for ingravescent cardiac failure. Echocardiographic examination (Fig. 2A) documented the presence of a recurred mass occupying almost totally the right ventricle. Total-body computer tomography exam did not reveal any other extracardiac mass. The patient died a few days after admission. Autopsy confirmed the absence of any other extracardiac mass and that the right ventricle was completely occupied by an infiltrative, white-yellowish tumor with hemorrhagic and necrotic areas (Fig. 2B). Microscopically (Fig. 2C), the tumor showed highly cellular and pleomorphic areas with large hyperchromatic spindle and epithelioid sometimes multinucleated cells, necrosis, and abundant mitoses. The diagnosis of high-grade pleomorphic sarcoma was made. Genomic polymerase chain reaction (PCR) of genomic DNA extracted from formalin-fixed, paraffinembedded tissue revealed FUS/Creb3L2 fusion gene in both primary and recurrent tumors (Fig. 2D).

\section{Comments}

Primary malignant sarcomas of the heart are extremely uncommon [1]. LGFMS is a distinctive variant of fibrosarcoma of soft tissues described for the first time by Evans in 1987 [2]. According to our knowledge, this is the first described case of primary LGFMS of the heart. In 2008, Jakowski and Wakely [3] described a case of intrathoracic LGFMS as a mass attached to the external epicardium, with no myocardial involvement. LGFMS should be considered in the 

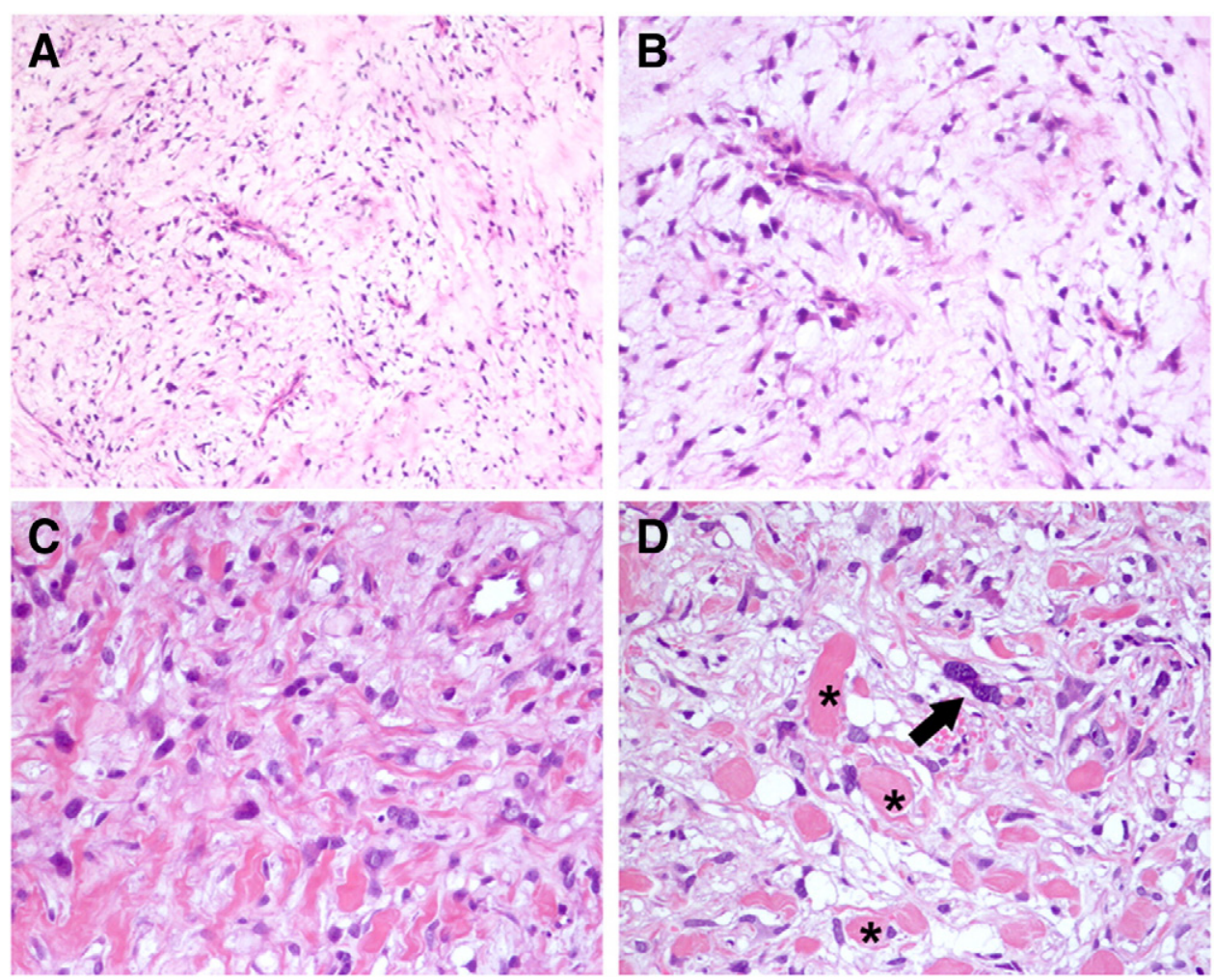

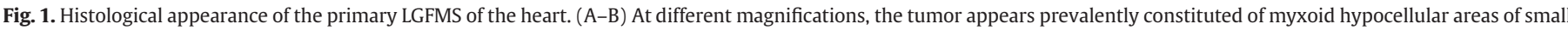


infiltration; residual cardiomyocytes (*). (A-D: hematoxylin and eosin stain; original magnification: A, 200×; B-D, 400×).

differential diagnosis with other primary myxoid cardiac tumors, including myxoma, low-grade myxoid fibrosarcoma or myxofibrosarcoma, and myxoid liposarcoma [1]. Myxoma is characterized by typical "ring structures," the absence of mitosis, and necrosis. Myxoid liposarcoma showed distinctive delicate chicken-wire capillary arborizing vasculature and, in the majority of cases, a diffuse S100
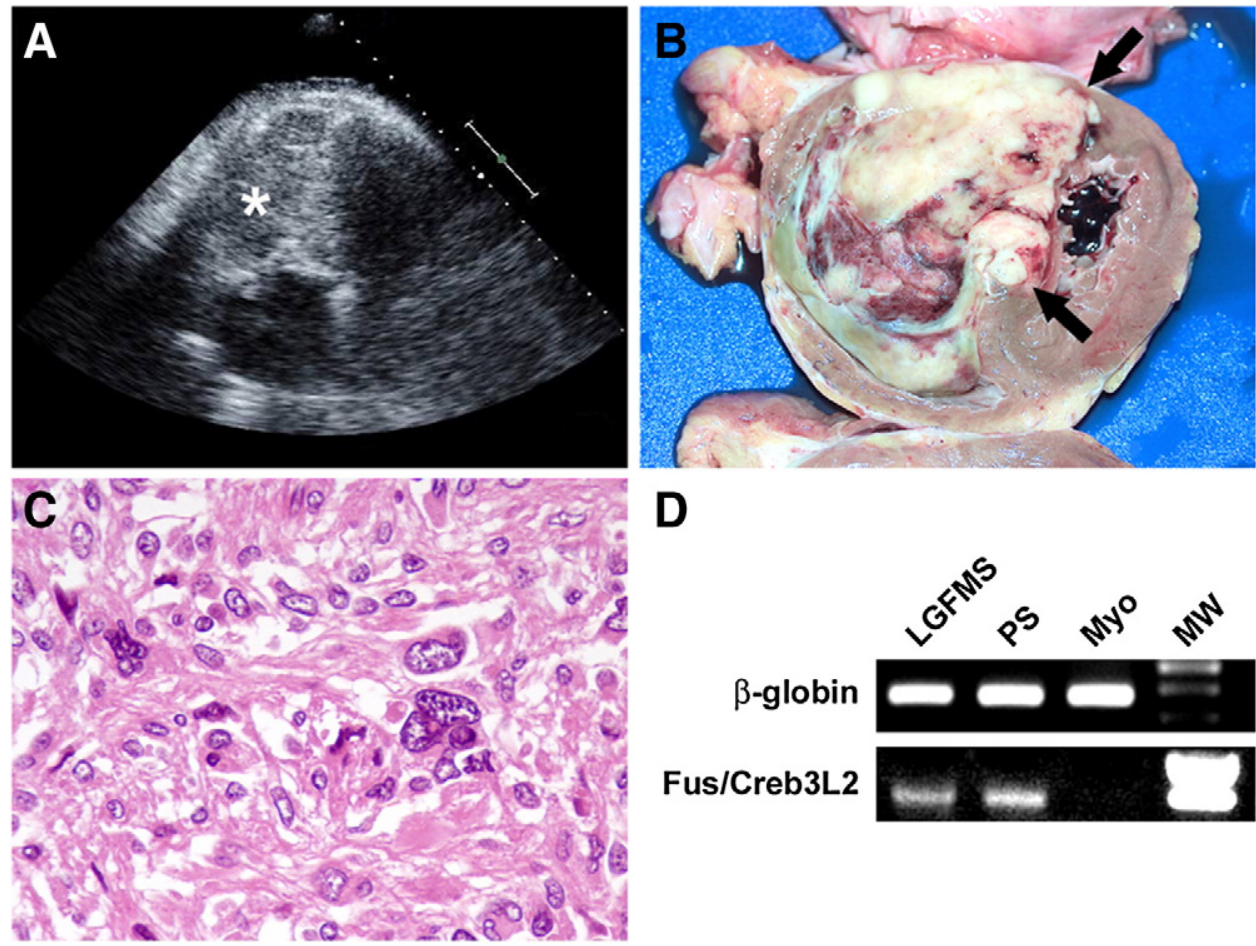

D



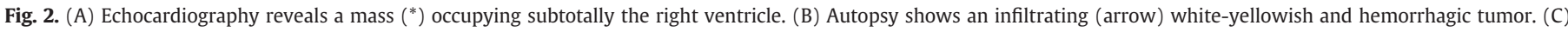

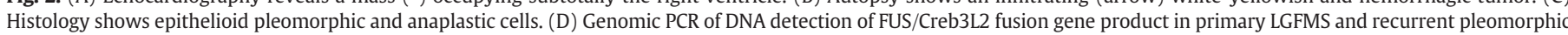
sarcoma (PS). Control ventricular myocardium (Myo) is negative. (A-D: hematoxylin and eosin stain; original magnification: C, 400×). 
immunopositivity. Myxofibrosarcoma lacks the typical alternation of myxoid and collagenous areas, and tumor cells are more spindle and atypical than in LGFMS. The "hyalinizing spindle cell tumor with giant rosettes" is now considered a variant of LGFMS since tumors share the specific translocation between chromosome bands 7q3334 (CREB3L2) and 16p11 (FUS) [4]. So, reverse transcriptase PCR or fluorescence in situ hybridization analysis of the chimeric FUS/ CREB3L2 may be used for differential diagnosis.

Soft-tissue LGFMS is an indolent tumor, typically occurring in the lower limb/groin area with a relatively benign appearance and, even with metastasis, a more favorable course than myxofibrosarcomas. In Evan's LGFMS series, one case displayed anaplastic dedifferentiation at 30-years follow-up [5]. The presence of a rich and delicate vascularization of LGFMS suggests a potential target for additional postsurgical therapy [6]. The most interesting features of this case are the first description of the cardiac location of an LGFMS, usually occurring in the lower limbs, and the progression after 7 years to a high-grade sarcoma. In fact, LGFMS of soft tissues has a deceptively benign appearance, with common recurrences and a low metastatic potential [2]. The recognition and diagnosis of cardiac LGFMS appear particularly relevant for the long survival rate compared to other sarcomas.

\section{References}

[1] Orlandi A, Ferlosio A, Roselli M, Chiariello L, Spagnoli LG. Cardiac sarcomas: an update. J Thorac Oncol 2010;5:1483-9.

[2] Vernon SE, Bejarano PA. Low-grade fibromyxoid sarcoma: a brief review. Arch Pathol Lab Med 2006;130:1358-60.

[3] Jakowski JD, Wakely PE. Primary intrathoracic low-grade fibromyxoid sarcoma. Hum Pathol 2008;39:623-8.

[4] Panagopoulos I, Storlazzi CT, Fletcher CD, Fletcher JA, Nascimento A, Domanski HA Wejde J, Brosjö O, Rydholm A, Isaksson M, Mandahl N, Mertens F. The chimeric FUS/CREB312 gene is specific for low-grade fibromyxoid sarcoma. Genes Chromosomes Cancer 2004; 40:218-28.

[5] Evans HL. Low-grade fibromyxoid sarcoma: a report of two metastasising neoplasms having a deceptively benign appearance. Am J Clin Pathol 1987;88: 615-9.

[6] Cassinelli G, Zuco V, Petrangolini G, De Cesare M, Tortoreto M, Lanzi C, Cominetti D, Zaffaroni N, Orlandi A, Passeri D, Meco D, Di Francesco AM, Riccardi R, Bucci F, Pisano C, Zunino F. The curative efficacy of namitecan (ST1968) in preclinical models of pediatric sarcoma is associated with antiangiogenic effects. Biochem Pharmacol 2012;84:163-71. 